\title{
On Anti-Periodic Solutions for a Class of Impulsive Retarded Functional Differential Equations
}

\author{
S. M. Afonso and A. L. Furtado
}

\begin{abstract}
In this paper, some new results on the existence and uniqueness of anti-periodic solution for a first-order impulsive functional differential equation with delay are obtained. The main tool to be employed here is the coincidence degree theory.
\end{abstract}

Mathematics Subject Classification. Primary 34K45; Secondary 47H11.

Keywords. Existence and uniqueness, anti-periodic solution, impulsive retarded differential equations, coincidence degree.

\section{Introduction}

Anti-periodic problems arise naturally from the mathematical models of a variety of physical processes (see $[1,2,11,17]$ ) and also appear in the study of differential equations (see $[4,5,7,14]$ ). For this reason, solutions to antiperiodic problems have been investigated by many authors in the last years. For instance, Chen et al. in [6] studied an anti-periodic problem for a nonlinear evolution equation, where the nonlinear part is an odd maximal monotone mapping. Liu and Migorski in [12] constructed a new and important existence result and analyzed the controllability for differential inclusions with anti-periodic conditions in Banach spaces, which was a major development in anti-periodic problems. Okochi [15] studied the existence of anti-periodic solutions to evolution equations of subdifferential type in Hilbert space.

Recently, Afonso and Furtado [3] applied the coincidence degree theory to analyze the existence and uniqueness of anti-periodic solution for the $n$ thorder functional differential equation with infinite delay:

$$
x^{(n)}(t)=f\left(t, x_{t}^{(n-1)}, x_{t}^{(n-2)}, \ldots, x_{t}^{\prime}, x_{t}\right), \quad t \in \mathbb{R} .
$$

This work was supported by Grant 2014/14607-7, São Paulo Research Foundation (FAPESP). 
The present work deals with a first-order impulsive retarded functional differential equation. To find sufficient conditions for the existence and uniqueness of anti-periodic solution for such equation, we make use of a non-impulsive equation conveniently associate with this. The same strategy was utilized in [13], by Meili et al., to guarantee the existence of periodic solution for an impulsive differential equation with discrete delay.

To exhibit the problem, we begin by recalling the concept of regulated functions. According to [8], we say that a function $\phi: I \rightarrow \mathbb{R}$, defined in a interval $I \subset \mathbb{R}$, is regulated when the following assertions holds:

$$
\text { if } \tau \text { is a left limit point, then } \lim _{t \rightarrow \tau^{-}} \phi(t) \in \mathbb{R}
$$

and

$$
\text { if } \tau \text { is a right limit point, then } \lim _{t \rightarrow \tau^{+}} \phi(t) \in \mathbb{R} \text {. }
$$

In this work, $G^{-}(I, \mathbb{R})$ denotes the set of the regulated functions $\phi: I \rightarrow \mathbb{R}$ which are continuous from the left.

We will adopt the following notations: given a non-negative constant $r$, then for each $t \geq 0$ and each function $x: I \rightarrow \mathbb{R}$ defined on a interval $I \subset \mathbb{R}$ which contains $[t-r, t]$, the symbol $x_{t}$ denotes the function $x_{t}:[-r, 0] \rightarrow \mathbb{R}$ given by $x_{t}(w)=x(t+w)$ for every $w \in[-r, 0]$.

Given a positive number $T$, we say that a continuous function $x: I \rightarrow \mathbb{R}$ is $T$-anti-periodic on $I \subset \mathbb{R}$ if

$$
x(t+T)=x(t) \text { and } x\left(t+\frac{T}{2}\right)=-x(t)
$$

for all $t \in \mathbb{R}$, such that $t+T \in I$.

Let $T, r>0$ and $f:[0,+\infty) \times G^{-}([-r, 0], \mathbb{R}) \rightarrow \mathbb{R}$ a continuous function $T$-periodic in the first argument, such that

(H) $f\left(t+\frac{T}{2},-\varphi\right)=-f(t, \varphi), \quad$ for $\varphi \in G^{-}([-r, 0], \mathbb{R})$ and $t \in[0,+\infty)$. Consider the following impulsive retarded differential equation:

$$
\begin{cases}x^{\prime}(t)=f\left(t, x_{t}\right), & t \geq 0, \quad t \neq t_{k}, \quad k=1,2,3, \ldots \\ x\left(t_{k}^{+}\right)=\left(1+b_{k}\right) x\left(t_{k}\right), & k=1,2,3, \ldots,\end{cases}
$$

where the numbers $b_{k}$ satisfy $\left(-1, \frac{T}{2}\right) \cap\left\{b_{k} ; k=1,2,3, \ldots\right\}=\left\{b_{1}, \ldots, b_{i}\right\}$, $b_{1}<b_{2}<\ldots<b_{i}$ and $b_{i+k}=\frac{T}{2}+b_{k}, k=1,2,3, \ldots$ The numbers $t_{k}$ are called instants (or moments) of impulse and satisfy $\left(0, \frac{T}{2}\right) \cap\left\{t_{k} ; k=\right.$ $1,2, \ldots\}=\left\{t_{1}, \ldots, t_{m}\right\}, t_{1}<t_{2}<\ldots<t_{m}$ and $t_{m+k}=\frac{T}{2}+t_{k}, k=1,2,3, \ldots$ Finally, $x\left(t_{k}^{+}\right)$denotes the right $\operatorname{limit}_{\lim } \operatorname{lit}_{k}^{+} x(t)$.

Definition 1.1. A function $x:[-r,+\infty) \rightarrow \mathbb{R}$ is said to be solution of problem (1.1) on $[0,+\infty)$ if the following conditions are satisfied:

(i) $x$ is absolutely continuous on each interval $\left[0, t_{1}\right],\left(t_{k}, t_{k+1}\right], k=1,2, \ldots$.

(ii) $x$ satisfies the first equality in (1.1) on $[0,+\infty) \backslash\left\{t_{k} ; k=1,2, \ldots\right\}$.

(iii) $x\left(t_{k}^{+}\right)=\left(1+b_{k}\right) x\left(t_{k}\right)$, for $k=1,2, \ldots$

A solution of (1.1) is said to be $T$-anti-periodic if its restriction to the interval $[0,+\infty)$ is $T$-anti-periodic. 
Let us consider the following assumption:

(A) There is a positive constant $a$, such that $\frac{a T}{2}<1$ and

$$
|f(t, \varphi)-f(t, \psi)| \leq a|\varphi(0)-\psi(0)|,
$$

for each $t \in[0,+\infty)$ and $\varphi, \psi \in G^{-}([-r, 0], \mathbb{R})$.

The text is organized as follows. In Sect. 1, we show that the analysis of the question of existence of a $T$-anti-periodic solution for problem (1.1) can be reduced to the study of the same question concerning a certain non-impulsive equation conveniently associated to it. In Sect. 2, we give sufficient conditions for the existence of a $T$-anti-periodic solution of class $C^{1}$ on $\left[0, t_{1}\right],\left(t_{k}, t_{k+1}\right]$, $k=1,2,3, \ldots$ for impulsive problem (1.1). The uniqueness of such $T$-antiperiodic solution for that problem is proved in Sect. 3. Finally, in Sect. 4, an example is given to demonstrate the effectiveness of the obtained results.

\section{Preliminaries}

Let $\beta:[-r,+\infty) \rightarrow \mathbb{R}$ be a function defined by

$$
\beta(t)= \begin{cases}1, & t \in\left[-r, t_{1}\right] \\ \prod_{t_{k}<t}\left(1+b_{k}\right) & t \in\left(t_{1}, \frac{T}{2}\right] \\ \beta\left(t-\frac{T}{2}\right), & t \in\left(\frac{T}{2},+\infty\right) .\end{cases}
$$

Define a function $h:[0,+\infty) \times G^{-}([-r, 0], \mathbb{R}) \rightarrow \mathbb{R}$ by

$$
h(t, \varphi)=\frac{f\left(t, \beta_{t} \varphi\right)}{\beta(t)}
$$

and consider the non-impulsive retarded equation:

$$
x^{\prime}(t)=h\left(t, x_{t}\right), \quad t \geq 0 .
$$

Definition 2.1. A function $x:[-r,+\infty) \rightarrow \mathbb{R}$ is said to be solution of Eq. (2.1) if the following conditions are satisfied:

(i) $x$ is absolutely continuous on $[0,+\infty)$.

(ii) $x$ satisfies the equality in (2.1) for almost everywhere $t \in[0,+\infty)$.

A solution of (2.1) is said to be $T$-anti-periodic if its restriction to the interval $[0,+\infty)$ is $T$-anti-periodic.

We claim that hypothesis $(H)$ and assumption $(A)$ on the function $f$ imply similar conditions on the function $h$. In fact, by $(H)$, we have

$$
\begin{aligned}
h\left(t+\frac{T}{2},-\varphi\right) & =\frac{f\left(t+\frac{T}{2},-\beta_{t+\frac{T}{2}} \varphi\right)}{\beta\left(t+\frac{T}{2}\right)} \\
& =\frac{f\left(t+\frac{T}{2},-\beta_{t} \varphi\right)}{\beta(t)} \\
& =-\frac{f\left(t, \beta_{t} \varphi\right)}{\beta(t)} \\
& =-h(t, \varphi),
\end{aligned}
$$


and from assumption $(A)$, it follows that

$$
\begin{aligned}
|h(t, \varphi)-h(t, \psi)| & =\left|\frac{f\left(t, \beta_{t} \varphi\right)}{\beta(t)}-\frac{f\left(t, \beta_{t} \psi\right)}{\beta(t)}\right| \\
& \leq a\left|\frac{\beta_{t}(0) \varphi(0)}{\beta(t)}-\frac{\beta_{t}(0) \psi(0)}{\beta(t)}\right| \\
& =a|\varphi(0)-\psi(0)| .
\end{aligned}
$$

The following result establishes the relation between $T$-anti-periodic solutions of impulsive problem (1.1) and non-impulsive equation (2.1), under the hypothesis that one of them has a $T$-anti-periodic solution. The importance of this result lies in the fact that it is possible to conclude that the impulsive problem (1.1) has a unique $T$-anti-periodic solution if non-impulsive equation (2.1) has this property.

Theorem 2.2. Problem (1.1) has a unique T-anti-periodic solution if, and only if, the same occurs with Eq. (2.1).

Proof. Suppose that $x:[-r,+\infty) \rightarrow \mathbb{R}$ is a $T$-anti-periodic solution of problem (1.1). Define a function $u:[-r,+\infty) \rightarrow \mathbb{R}$ by $u(t)=x(t) / \beta(t)$. Note that, for $t \in[0,+\infty)$

$$
\begin{aligned}
u(t+T) & =\frac{x(t+T)}{\beta(t+T)}=\frac{x(t)}{\beta(t)}=u(t) \quad \text { and } \\
u\left(t+\frac{T}{2}\right) & =\frac{x\left(t+\frac{T}{2}\right)}{\beta\left(t+\frac{T}{2}\right)}=-\frac{x(t)}{\beta(t)}=-u(t),
\end{aligned}
$$

since $x$ is $T$-anti-periodic and $\beta$ is $\frac{T}{2}$-periodic on $[0,+\infty)$. Therefore, $u$ is $T$-anti-periodic on $[0,+\infty)$.

Moreover, since $x$ and $\beta$ are continuous on each interval $\left[0, t_{1}\right],\left(t_{k}, t_{k+1}\right]$, $k=1,2,3, \ldots$, then the function $u$ has the same property. To conclude that $u$ is continuous on $[0,+\infty)$, it is enough to prove that it is continuous from the right at each impulsive moment $t_{k}$. In fact, this occurs, because $x$ satisfies the first equality of (1.1), and then, for each $k=1,2, \ldots$, we have

$$
\begin{aligned}
\lim _{t \rightarrow t_{k}^{+}} u(t) & =\lim _{t \rightarrow t_{k}^{+}} x(t) / \beta(t)=\lim _{t \rightarrow t_{k}^{+}} x(t)\left[\prod_{t_{j}<t}\left(1+b_{j}\right)^{-1}\right] \\
& =x\left(t_{k}^{+}\right) \prod_{t_{j} \leq t_{k}}\left(1+b_{j}\right)^{-1}=\left(1+b_{k}\right) x\left(t_{k}\right)\left(1+b_{k}\right)^{-1} \prod_{t_{j}<t_{k}}\left(1+b_{j}\right)^{-1} \\
& =\frac{x\left(t_{k}\right)}{\beta\left(t_{k}\right)}=u\left(t_{k}\right) .
\end{aligned}
$$

Let us verify that the function $u$ satisfies the equality given in (2.1) for almost everywhere $t \in[0,+\infty)$. Using the hypothesis that $x$ is solution of problem (1.1) and the definition of function $h$, we obtain 


$$
\begin{aligned}
u^{\prime}(t) & =\frac{x^{\prime}(t)}{\beta(t)} \\
& =\frac{f\left(t, x_{t}\right)}{\beta(t)}=\frac{f\left(t, \beta_{t} u_{t}\right)}{\beta(t)} \\
& =h\left(t, u_{t}\right),
\end{aligned}
$$

for almost every $t \in[0,+\infty)$. This completes the proof that $u$ is a $T$-antiperiodic solution of problem (2.1).

We have to prove that the uniqueness of $T$-anti-periodic solution for nonimpulsive equation (2.1) implies the uniqueness of $T$-anti-periodic solution to impulsive problem (1.1). In fact, assume that Eq. (2.1) has at most one $T$-anti-periodic solution. If problem (1.1) had two distinct $T$-anti-periodic solutions, $x(t)$ and $\widehat{x}(t)$, then, by what we have proved above, the functions $u(t)=x(t) / \beta(t)$ and $\widehat{u}(t)=\widehat{x}(t) / \beta(t)$ would be two distinct $T$-anti-periodic solutions for Eq. (2.1), which contradicts the assumption. Therefore, if Eq. (2.1) has at most one $T$-anti-periodic solution, the same occurs with problem (1.1).

Now, suppose that $u$ is a $T$-anti-periodic solution of non-impulsive equation (2.1). Let us show that the function $x:[-r,+\infty) \rightarrow \mathbb{R}$ given by $x(t)=\beta(t) u(t)$ is a $T$-anti-periodic solution of problem (1.1). The $T$-antiperiodicity of function $x$ on $[0,+\infty)$ follows immediately from the fact that $u$ has such property and $\beta$ is $\frac{T}{2}$-periodic. Note that function $x$ satisfies the conditions from Definition 1.1. Indeed, since $u$ and $\beta$ are absolutely continuous on each interval $\left[0, t_{1}\right],\left(t_{k}, t_{k+1}\right], k=1,2,3, \ldots$, then the function $x$ is also absolutely continuous on these intervals. Furthermore, since $u$ is solution of Eq. (2.1), we have

$$
\begin{aligned}
x^{\prime}(t)-f\left(t, x_{t}\right) & =\beta(t) u^{\prime}(t)-f\left(t, \beta_{t} u_{t}\right)=\beta(t)\left[u^{\prime}(t)-\frac{f\left(t, \beta_{t} u_{t}\right)}{\beta(t)}\right] \\
& =\beta(t)\left[u^{\prime}(t)-h\left(t, u_{t}\right)\right]=0,
\end{aligned}
$$

for almost every $t \in[0,+\infty) \backslash\left\{t_{1}, t_{2} \ldots\right\}$.

We claim that $x\left(t_{k}^{+}\right)=\left(1+b_{k}\right) x\left(t_{k}\right)$, for $k=1,2,3, \ldots$ In fact, for each $k=1,2,3 \ldots$, we have

$$
\begin{aligned}
x\left(t_{k}^{+}\right) & =\lim _{t \rightarrow t_{k}^{+}} \beta(t) u(t)=\lim _{t \rightarrow t_{k}^{+}}\left[\prod_{t_{i}<t}\left(1+b_{i}\right) u(t)\right] \\
& =\prod_{t_{i} \leq t_{k}}\left(1+b_{i}\right) u\left(t_{k}\right)=\left(1+b_{k}\right) \beta\left(t_{k}\right) u\left(t_{k}\right) \\
& =\left(1+b_{k}\right) x\left(t_{k}\right) .
\end{aligned}
$$

Thus, if $u$ is a $T$-anti-periodic solution of non-impulsive equation (2.1), the function $x: t \mapsto u(t) \beta(t), t \in[-r,+\infty)$, is a $T$-anti-periodic solution of impulsive problem (1.1).

To finalize the proof of this lemma, assume that problem (1.1) has at most one $T$-anti-periodic solution. By what we have seen above, if Eq. (2.1) had two distinct $T$-anti-periodic solutions, $u(t)$ and $\widehat{u}(t)$, then problem (1.1) would have also two distinct $T$-anti-periodic solutions $x(t)=u(t) \beta(t)$ and 
$\widehat{x}(t)=\widehat{u}(t) \beta(t)$, which contradicts our assumption. Therefore, if problem (1.1) has at most one $T$-anti-periodic solution, the same occurs with Eq. (2.1).

Remark 2.3. Note that of the demonstration of Theorem 2.2, we can infer that if non-impulsive equation (2.1) has a solution of class $C^{1}$ on $[0,+\infty)$, then the impulsive problem (1.1) has a solution of class $C^{1}$ on the intervals $\left[0, t_{1}\right],\left(t_{k}, t_{k+1}\right], k=1,2,3, \ldots$

For the sake of overcoming the difficulties arising from impulsive effects present in problem (1.1), we will use Theorem 2.2 to obtain sufficient conditions for existence and uniqueness of a $T$-anti-periodic solution for it through of analysis of problem of existence and uniqueness of a $T$-anti-periodic solution for non-impulsive equation (2.1). In the next section, we will study the existence of a such solution.

\section{Existence of Anti-periodic Solution}

Our purpose in this section is to prove the following result:

Theorem 3.1. If assertion $(A)$ is fulfilled, then problem (1.1) has at least one $T$-anti-periodic solution which is of class $C^{1}$ on each interval $\left[0, t_{1}\right],\left(t_{k}, t_{k+1}\right]$, $k=1,2,3, \ldots$.

Our strategy to prove Theorem 3.1 demonstrates that if condition $(A)$ is fulfilled, then Eq. (2.1) has at least one $T$-anti-periodic solution which is of class $C^{1}$ on $[0,+\infty)$. By Theorem 2.2 and Remark 2.3 , we can verify the validity of Theorem 3.1 .

Theorem 3.2. If condition $(A)$ is fulfilled, then Eq. (2.1) has at least one $T$-anti-periodic solution which is of class $C^{1}$ on $[0,+\infty)$.

To prove Theorem 3.2, we start by recalling some concepts concerning at the next lemma, which is crucial in the arguments of this section.

Let $X$ and $Y$ be Banach spaces. We say that a linear operator $L$ : $\operatorname{Dom} L \subset X \rightarrow Y$ is a Fredholm operator if $\operatorname{Ker} L=\{x \in X ; L x=0\}$ is finitedimensional and $\operatorname{Im} L=\{L(x) \in Y ; x \in X\}$ is closed in $Y$ and is of finite codimension on $Y$. The index of $L$ is defined by $\operatorname{dim} \operatorname{Ker} L-\operatorname{codim} \operatorname{Im} L$. It is possible to prove (see [9]) that if $L$ is a Fredholm operator of index zero, then there exist continuous linear and idempotent operators $P: X \rightarrow X$ and $Q: Y \rightarrow Y$, such that

$$
\operatorname{Ker} L=\operatorname{Im} P \quad \text { and } \quad \operatorname{Im} L=\operatorname{Ker} Q .
$$

The first equality in (3.1) implies that the restriction of $L$ to $\operatorname{Dom} L \cap \operatorname{Ker} P$, which we will denote by $L_{P}$, is an isomorphism onto its image. Indeed, by supposing $\operatorname{Ker} L=\operatorname{Im} P$ and taking $x \in \operatorname{Dom} L \cap \operatorname{Ker} P$, such that $L_{P}(x)=0$, we have that $x \in \operatorname{Im} P$, that is, there exists $y \in X$, such that $P y=x$. Since $P$ is idempotent and $x \in \operatorname{Ker} P$, the last equality implies $x=P y=P x=0$.

By assuming that $L: \operatorname{Dom} L \subset X \rightarrow Y$ is a Fredholm operator of index zero and $P$ and $Q$ are the aforementioned operators, we say that a 
continuous operator $N: X \rightarrow Y$ is $L$-compact on $\bar{\Omega}$, where $\Omega \subset X$ is open and bounded and $\bar{\Omega}$ is its closure if $Q N(\bar{\Omega})$ is bounded and the operator $\left(L_{P}\right)^{-1}(I-Q) N: \bar{\Omega} \rightarrow X$ is compact.

The proof of the following lemma can be found in [16].

Lemma 3.3. Let $X, Y$ be Banach spaces, $\Omega \subset X$ a bounded open set symmetric with $0 \in \Omega$. Suppose $L: \operatorname{Dom} L \subset X \rightarrow Y$ is a Fredholm operator of index zero with $\operatorname{Dom} L \cap \bar{\Omega} \neq \emptyset$ and $N: X \rightarrow Y$ is a L-compact operator on $\bar{\Omega}$. Assume, moreover, that

$$
L x-N x \neq-\lambda(L x+N(-x)),
$$

for all $x \in \operatorname{Dom} L \cap \partial \Omega$ and all $\lambda \in(0,1]$, where $\partial \Omega$ is the boundary of $\Omega$ with respect to $X$. Under these conditions, the equation $L x=N x$ has at least one solution on $\operatorname{Dom} L \cap \bar{\Omega}$.

In the sequel, we will construct an equation $L x=N x$ that appropriately mirror equation (2.1), so that all the conditions of Lemma 3.3 are fulfilled.

We will adopt the following notations:

$$
\begin{gathered}
C_{T}^{k}=\left\{x \in C^{k}([0,+\infty), \mathbb{R}) ; x \quad \text { is } T \text {-periodic }\right\}, \quad k \in\{0,1\}, \\
\|x\|_{2}=\left(\int_{0}^{T}|x(t)|^{2} \mathrm{~d} t\right)^{1 / 2}, \quad\|x\|_{\infty}=\max _{t \in[0, T]}|x(t)|, \quad \text { for } x \in C_{T}^{0}, \\
\left\|x^{\prime}\right\|_{\infty}=\max _{t \in[0, T]}\left|x^{\prime}(t)\right|, \quad \text { for } x \in C_{T}^{1} .
\end{gathered}
$$

Define the sets

$$
X=\left\{x \in C_{T}^{1} ; x\left(t+\frac{T}{2}\right)=-x(t), \quad t \in[0,+\infty)\right\}
$$

and

$$
Y=\left\{x \in C_{T}^{0} ; x\left(t+\frac{T}{2}\right)=-x(t), \quad t \in[0,+\infty)\right\} .
$$

By equipping $X$ and $Y$, respectively, with the norms $\|\cdot\|_{X}$ and $\|\cdot\|_{Y}$, given by

$$
\|x\|_{X}=\max \left\{\|x\|_{\infty},\left\|x^{\prime}\right\|_{\infty}\right\},\|x\|_{Y}=\|x\|_{\infty},
$$

we obtain two Banach spaces that, for simplicity of notation, we will denote them by $X$ and $Y$, respectively.

Define the operators $L: X \rightarrow Y$ and $N: X \rightarrow Y$ by

$$
L x(t)=x^{\prime}(t), \quad t \in[0,+\infty),
$$

and

$$
N x(t)=h\left(t, x_{t}\right), \quad t \in[0,+\infty) .
$$


Remark 3.4. Note that, if the equation

$$
L x(t)=N x(t), \quad[0, T]
$$

has a solution, then problem (2.1) has a $T$-anti-periodic solution which is of class $C^{1}$ on $[0,+\infty)$. Indeed, if $\hat{x}$ is a solution of $(3.4)$ on $[0, T]$, it is enough to define $x:[0,+\infty) \rightarrow \mathbb{R}$ by $x(t)=\hat{x}(t)$ if $t \in(0, T]$ and $x(t)=x(t-T)$ if $t \in(T,+\infty)$.

To prove Theorem 3.2, we will show that if condition $(A)$ is fulfilled, then the hypotheses of Lemma 3.3 are satisfied for $L$ and $N$ defined above, and consequently, the result will follow from Theorem 2.2 and Remark 3.4.

Now, we can prove the following result.

Proposition 3.5. The operator $L$ defined in (3.2) is a Fredholm operator of index zero.

Proof. We claim that $\operatorname{Im} L=Y$. Indeed, given $y \in Y$, let

$$
x(t)=-\frac{1}{2} \int_{t}^{t+\frac{T}{2}} y(s) \mathrm{d} s .
$$

Clearly, $x \in X$ and $L x(t)=y(t)$. Furthermore, it is easy to see that $\operatorname{Ker} L=0$. Then, the proof is complete.

Proposition 3.6. The operator $N$ is L-compact on any bounded open set $\Omega \subset$ $X$.

Proof. Let us consider the operators $P$ and $Q$ given by

$$
P x=\frac{1}{T} \int_{0}^{T} x(t) \mathrm{d} t, x \in X \quad \text { and } \quad Q y=\frac{1}{T} \int_{0}^{T} y(t) \mathrm{d} t, \quad y \in Y .
$$

Thus, $\operatorname{Im} P=\operatorname{Ker} L$ and $\operatorname{Ker} Q=\operatorname{Im} L$. Denote by $L_{P}^{-1}: \operatorname{Im} L \rightarrow X \cap \operatorname{Ker} P$, the inverse of $L_{\mid X \cap \operatorname{Ker} P}$. Then

$$
\left(L_{P}^{-1} z\right)(t)=\int_{0}^{t} z(s) \mathrm{d} s-\frac{1}{T} \int_{0}^{T}\left(\int_{0}^{t} z(s) \mathrm{d} s\right) \mathrm{d} \tau .
$$

One can observe that $L_{P}^{-1}$ is a compact operator. Furthermore, it is not difficult to show that, for any open bounded set $\Omega \subset X$, the set $Q N(\bar{\Omega})$ is bounded, and using the Arzelà-Ascoli's Theorem, the operator $L_{P}^{-1}(I-Q) N$ : $\bar{\Omega} \rightarrow X$ is compact. Therefore, $N$ is L-compact on $\Omega$.

The next lemma will be useful in the sequence. Its demonstration can be found in [10].

Lemma 3.7. If $v: \mathbb{R} \rightarrow \mathbb{R}$ is a T-periodic absolutely continuous function, such that $\int_{0}^{T} v(t) \mathrm{d} t=0$ and $\int_{0}^{T} v^{\prime}(t)^{2} \mathrm{~d} t \in \mathbb{R}$, then

$$
\int_{0}^{T} v(t)^{2} \mathrm{~d} t \leq \frac{T^{2}}{4 \pi^{2}} \int_{0}^{T} v^{\prime}(t)^{2} \mathrm{~d} t
$$


Proposition 3.8. If condition $(A)$ holds, then there exist a positive number $D$, which does not depend on $\lambda$, such that if

$$
L x-N x=-\lambda[L x+N(-x)], \quad \lambda \in(0,1],
$$

then $\|x\|_{X} \leq D$.

Proof. Assume that condition $(A)$ is satisfied and that $x \in X$ satisfies (3.5). Then, using the definitions of operators $L$ and $N$, given in (3.2) and (3.3), respectively, we obtain

$$
x^{\prime}(t)=\frac{1}{1+\lambda} h\left(t, x_{t}\right)-\frac{\lambda}{1+\lambda} h\left(t,-x_{t}\right) .
$$

Thereby, considering $H(t, x)=h\left(t, x_{t}\right)$, we have

$$
x^{\prime}(t)=\frac{1}{1+\lambda} H(t, x)-\frac{\lambda}{1+\lambda} H(t,-x) .
$$

Multiplying both sides of this equality by $x^{\prime}(t)$ and subsequently integrating it from 0 to $T$ and using the triangle inequality, we get

$$
\begin{aligned}
\left\|x^{\prime}\right\|_{2}^{2} \leq & \frac{1}{1+\lambda} \int_{0}^{T}|H(t, x)|\left|x^{\prime}(t)\right| \mathrm{d} t+\frac{\lambda}{1+\lambda} \int_{0}^{T}\left|H(t,-x) \| x^{\prime}(t)\right| \mathrm{d} t \\
\leq & \frac{1}{1+\lambda}\left[\int_{0}^{T}|H(t, x)-H(t, 0)|\left|x^{\prime}(t)\right| \mathrm{d} t+\int_{0}^{T}|H(t, 0)|\left|x^{\prime}(t)\right| \mathrm{d} t\right] \\
& +\frac{\lambda}{1+\lambda}\left[\int_{0}^{T}|H(t,-x)-H(t, 0)|\left|x^{\prime}(t)\right| \mathrm{d} t+\int_{0}^{T}|H(t, 0)|\left|x^{\prime}(t)\right| \mathrm{d} t\right] .
\end{aligned}
$$

Therefore

$$
\begin{aligned}
\left\|x^{\prime}\right\|_{2}^{2} \leq & \int_{0}^{T} \max \{|H(t, x)-H(t, 0)|,|H(t,-x)-H(t, 0)|\}\left|x^{\prime}(t)\right| \mathrm{d} t \\
& +\int_{0}^{T}|H(t, 0)|\left|x^{\prime}(t)\right| \mathrm{d} t .
\end{aligned}
$$

This, assumption $(A)$, Hölder inequality and Lemma 3.7, imply

$$
\begin{aligned}
\left\|x^{\prime}\right\|_{2}^{2} & \leq a\left\|x^{\prime}\right\|_{2}\|x\|_{2}+R \sqrt{T}\left\|x^{\prime}\right\|_{2}, \\
& \leq \frac{a T}{2 \pi}\left\|x^{\prime}\right\|_{2}^{2}+R \sqrt{T}\left\|x^{\prime}\right\|_{2},
\end{aligned}
$$

where $R=\max _{t \in[0, T]}|H(t, 0)|$. Thus, we obtain

$$
\left\|x^{\prime}\right\|_{2} \leq K
$$

where $K=\frac{R \sqrt{T}}{1-\frac{a T}{2 \pi}}$, since $\frac{a T}{2 \pi}<1$ by assumption $(A)$.

By mean value theorem for integrals, we conclude that there exists $\tau_{j} \in$ $[0, T]$, such that $x^{\prime}\left(\tau_{j}\right)=0$, since $\int_{0}^{T} x^{\prime}(t) \mathrm{d} t=0$. Hence, by Hölder inequality, we have 


$$
|x(t)|=\left|\int_{\tau_{j}}^{t} x^{\prime}(s) \mathrm{d} s\right| \leq \int_{0}^{T}\left|x^{\prime}(s) \mathrm{d} s\right| \leq \sqrt{T}\left\|x^{\prime}\right\|_{2}, \quad t \in[0, T] .
$$

Consequently, $\|x\|_{\infty} \leq \sqrt{T}\left\|x^{\prime}\right\|_{2}$. Now, inequality (3.7) implies

$$
\|x\|_{\infty} \leq K \sqrt{T}
$$

Now note that, by (3.6), assumption $(A)$ and (3.9), we have

$$
\begin{aligned}
\left|x^{\prime}(t)\right| & \leq \max \{|H(t, x)-H(t, 0)|,|H(t,-x)-H(t, 0)|\}+\max _{t \in[0, T]}|H(t, 0)| \\
& \leq a|x(t)|+R \\
& \leq S,
\end{aligned}
$$

for all $t \in[0, T]$, where $S=a K \sqrt{T}+R$. Consequently

$$
\left\|x^{\prime}\right\|_{\infty} \leq S \text {. }
$$

Finally, by (3.9) and (3.10), we conclude that

$$
\|x\|_{X} \leq D
$$

where $D=\max \{K \sqrt{T}, S\}$.

Proposition 3.9. If condition $(A)$ is satisfied, then there is a bounded open set $\Omega \subset X$, such that

$$
L x-N x \neq-\lambda(L x+N(-x)),
$$

for all $x \in \partial \Omega$ and all $\lambda \in(0,1]$.

Proof. Assume that condition $(A)$ is satisfied. By Proposition 3.8, there exists a positive constant $D$, which does not depend on $\lambda$, such that if $x$ satisfies the equality $L x-N x=-\lambda(L x+N(-x)), \lambda \in(0,1]$, then $\|x\|_{X} \leq D$. Thus, if

$$
\Omega=\left\{x \in X ;\|x\|_{X}<M\right\},
$$

where $M>D$, we conclude that

$$
L x-N x \neq-\lambda(L x-N(-x)),
$$

for every $x \in \partial \Omega=\left\{x \in X ;\|x\|_{X}=M\right\}$ and $\lambda \in(0,1]$.

In this moment, we are ready to prove Theorem 3.2.

Proof of Theorem 3.2. Suppose $(A)$ holds. Clearly, the set $\Omega$ defined in (3.11) is symmetric, $0 \in \Omega$ and $X \cap \bar{\Omega}=\bar{\Omega} \neq \emptyset$. Furthermore, it follows from Proposition 3.9 that

$$
L x-N x \neq-\lambda[L x-N(-x)],
$$

for all $x \in X \cap \partial \Omega=\partial \Omega$ and all $\lambda \in(0,1]$. This, together with Lemma 3.3, implies that Eq. (2.1) has at least one $T$-anti-periodic solution.

To finish this section, we will prove Theorem 3.1.

Proof of Theorem 3.1. It follows immediately from Theorems 2.2, 3.2 and Remark 2.3. 


\section{Uniqueness of $T$-anti-periodic Solution}

Our goal in this section is to prove the following result:

Theorem 4.1. If condition $(A)$ is fulfilled, then problem (1.1) has at most one T-anti-periodic solution.

To demonstrate Theorem 4.1, we will show that if condition $(A)$ is fulfilled, then Eq. (2.1) has at most one $T$-anti-periodic solution. By Theorem 2.2 , this implies that problem (1.1) has at most one $T$-anti-periodic solution.

Theorem 4.2. If $(A)$ holds, then Eq. (2.1) has at most one T-anti-periodic solution.

Proof. Assume that condition $(A)$ is satisfied and that $x$ and $y$ are $T$-antiperiodic solutions of Eq. (2.1). To get the result, we have to show that the function $z=x-y$ is identically zero. Then, whereas $x$ and $y$ are $T$-periodic, it is sufficient to prove that $z(t)=0$ for all $t \in[0, T]$.

Since $x$ and $y$ are solutions of Eq. (2.1), then

$$
z^{\prime}(t)=h\left(t, x_{t}\right)-h\left(t, y_{t}\right) \text {. }
$$

Multiplying both sides of (4.1) by $z^{\prime}(t)$, integrating it from 0 to $T$, using hypothesis $(A)$ and Hölder inequality, we get

$$
\begin{aligned}
\left\|z^{\prime}\right\|_{2}^{2} & =\int_{0}^{T}\left|z^{\prime}(t) \| h\left(t, x_{t}\right)-h\left(t, y_{t}\right)\right| \mathrm{d} t \\
& \leq a \int_{0}^{T}\left|z^{\prime}(t) \| z(t)\right| \mathrm{d} t \\
& \leq a\left\|z^{\prime}\right\|_{2}\|z\|_{2} .
\end{aligned}
$$

On the other hand, by Lemma 3.7

$$
\|z\|_{2} \leq \frac{T}{2 \pi}\left\|z^{\prime}\right\|_{2}
$$

From this and (4.2), we obtain

$$
\left\|z^{\prime}\right\|_{2}^{2} \leq \frac{a T}{2 \pi}\left\|z^{\prime}\right\|_{2}^{2}
$$

Then, since $\frac{a T}{2 \pi}<1$, we have $\left\|z^{\prime}\right\|_{2}=0$. Besides, since $\|z\|_{\infty} \leq \sqrt{T}\left\|z^{\prime}\right\|_{2}$ (see (3.8)), we conclude that $z \equiv 0$ and the proof is complete.

Now, we can prove Theorem 4.1.

Proof. (of Theorem 4.1) It follows immediately from Theorems 2.2 and 4.2.

We complete our section with the following result.

Theorem 4.3. If assertion $(A)$ is fulfilled, then problem (1.1) has a unique $T$-anti-periodic solution of class $C^{1}$ on each interval $\left[0, t_{1}\right],\left(t_{k}, t_{k+1}\right], k=$ $1,2,3, \ldots$

Proof. The result follows straightaway from Theorems 3.1 and 4.2. 


\section{Example}

Suppose that, in problem (1.1), $r=1, T=2 \pi$, the numbers $b_{k}$ are given by

$$
b_{1}=2, \quad b_{2}=3, \quad b_{2+k}=\pi+b_{k}, \quad k=1,2,3, \ldots
$$

and the moments of impulse are

$$
t_{1}=1, \quad t_{2}=3 / 2, \quad t_{3}=5 / 2, \quad t_{3+k}=\pi+t_{k}, \quad k=1,2,3, \ldots
$$

With such data, consider the problem

$$
\left\{\begin{array}{l}
x^{\prime}(t)=f\left(t, x_{t}\right), \quad t \geq 0, \quad t \neq t_{k}, \quad k=1,2,3, \ldots \\
x\left(t_{k}^{+}\right)=\left(1+b_{k}\right) x\left(t_{k}\right),
\end{array}\right.
$$

where the function $f:[0,+\infty) \times G^{-}([-1,0], \mathbb{R}) \rightarrow \mathbb{R}$ is given by $f(t, \varphi)=$ $\frac{\sin t}{15} \varphi(0)$, for $t \in[0,+\infty)$ and $\varphi \in G^{-}([-1,0], \mathbb{R})$.

Note that if $\varphi \in G^{-}([-1,0], \mathbb{R})$, then

$$
f(t+\pi, \varphi)=-f(t, \varphi), \quad \text { for each } t \in[0,+\infty) .
$$

Let us see that, in this case, condition $(A)$ is satisfied. Indeed

(A) If $\varphi, \psi \in G^{-}([-1,0], \mathbb{R})$, we have

$$
|f(t, \varphi)-f(t, \psi)| \leq a|\varphi(0)-\psi(0)|,
$$

for each $t \in[0,+\infty)$, where $a=\frac{1}{15}$.

By Theorem 4.3, problem (5.1) has a unique $2 \pi$-anti-periodic solution of class $C^{1}$ on each interval $\left[0, t_{1}\right],\left(t_{k}, t_{k+1}\right], k=1,2,3, \ldots$

\section{Acknowledgements}

The authors would like to thank the referees for their valuable comments and suggestions which helped to improve the manuscript.

\section{References}

[1] Abdurahman, A., Anton, F., Bordes, J.: Half-string oscillator approach to string field theory (Ghost sector: I). Nuclear Phys. B 397, 260-282 (1993)

[2] Ahn, C., Rim, C.: Boundary flows in general coset theories. J. Phys. A 32, 2509-2525 (1999)

[3] Afonso, S.M., Furtado, A.L.: Antiperiodic solutions for nth-order functional differential equations with infinite delay. Electron. J. Differ. Equ. 44, 1-8 (2016)

[4] Aftabizadeh, A.R., Aizicovici, S., Pavel, N.H.: On a class of second-order antiperiodic boundary value problems. J. Math. Anal. Appl. 171, 301-320 (1992)

[5] Aizicovici, S., Pavel, N.H.: Anti-periodic solutions to a class of nonlinear differential equations in Hilbert space. J. Funct. Anal. 99, 387-408 (1991)

[6] Chen, Y.Q., Nieto, J.J., O'Regan, D.: Anti-periodic solutions for evolution equations associated with maximal monotone mappings. Appl. Math. Lett. 24, 302-307 (2011)

[7] Fan, Q.Y., Wang, W.T., Yi, X.J.: Anti-periodic solutions for a class of nonlinear nth-order differential equations with delays. J. Comput. Appl. Math. 230, 762769 (2009) 
[8] Fraňková, D.: Regulated functions. Math. Bohem. 1, 20-59 (1991)

[9] Gaines, R.E., Mawhin, J.: Coincidence Degree and Nonlinear Differential Equations. Lecture Notes in Math., vol. 568. Springer, Berlin (1977)

[10] Hardy, G.H., Littlewood, J.E., Pólya, G.: Inequalities. Cambridge University Press, London (1964)

[11] Kleinert, H., Chervyakov, A.: Functional determinants from Wronski Green function. J. Math. Phys. 40, 6044-6051 (1999)

[12] Liu, Z.H., Migorski, S.: Analysis and control of differential inclusions with antiperiodic conditions. Proc. R. Soc. Edinb. 144A(3), 457-467 (2014)

[13] Li, M., Kou, C., Duan, Y.: The existence of periodic solution of impulsive functional differential equation with infinite delay. J. Appl. Math. Comput. 29, 341-348 (2009)

[14] Okochi, H.: On the existence of anti-periodic solutions to nonlinear parabolic equations in noncylindrical domains. Nonlinear Anal. 14, 771-783 (1990)

[15] Okochi, H.: On the existence of anti-periodic solutions to a nonlinear evolution equation associated with odd subdifferential operators. J. Funct. Anal. 91, 246258 (1990)

[16] O' Regan, D., Chao, Y.J., Chen, Y.Q.: Topological Degree Theory and Application. Taylor and Francis Group, Boca Raton (2006)

[17] Pinsky, S., Tritman, U.: Antiperiodic boundary conditions to supersymmetric discrete light cone quantization. Phys. Rev. D 62, 087701 (2000)

\section{S. M. Afonso}

Departamento de Matemática

Instituto de Geociências e Ciências Exatas, UNESP - Univ Estadual Paulista

Avenida 24-A, 1515, Caixa Postal 178

Rio Claro SP13506-900

Brazil

e-mail: smafonso@rc.unesp.br

\section{A. L. Furtado}

Departamento de Análise Matemática

Instituto de Matemática e Estatística, Universidade do Estado do Rio de Janeiro

Rio de Janeiro RJ

Brazil

e-mail: andre.furtado@ime.uerj.br

Received: September 25, 2016.

Revised: March 25, 2017.

Accepted: July 21, 2017. 